\title{
A new insight into the molecular mechanism of thiazolidinediones in atrial fibrillation
}

\author{
Yanmin $\mathrm{Xu}^{*}$ and Shristi Dahal \\ International Medical School, Tianjin Medical University, No.22 Qi Xiang Tai Road, He Ping District, Tianjin 300070, P.R. China
}

Atrial fibrillation is the most frequent arrhythmia and incidence is increasing parallel to the aging of the population. As with all arrhythmias, a trigger delivered onto a susceptible substrate initiates the fibrillation. Facilitators adopt the substrate, allowing both the initiation and perpetuation of arrhythmia. However, the mechanism that adjusts the trigger to the substrate has not been completely elucidated.

According to Framingham Heart Study, men and women with diabetes mellitus have a $40 \%$ and $60 \%$ increased risk of atrial fibrillation (AF), respectively [1]. In the Atherosclerosis Risk in Communities study, AF risk is independently associated with HbA1c [2]. Insulin resistance alters cardiac structure via impaired mitochondrial function and oxidative stress that in turn modify transcriptional and translational processes essential for cardiac adaptation [1]. Examination of gene transcriptional profiles in human atrial tissue reveals a regulatory shift towards pro-oxidation in AF patients [3]. Atrial structural remodeling defined by extensive interstitial fibrosis is an important mechanism for AF development in diabetic heart [4]. Hyperglycemia contributes to atrial dilation and interstitial fibrosis resulting in interatrial conduction delay and hence, arrhythmogenicity [5]. Down-regulation of L-type calcium current (ICaL) and late sodium (INa) densities contributes to AF maintenance [4].

Certain clinical and demographic risk factors that link AF to $\mathrm{DM}$ are increasing age, female sex, whites, obesity, hypertension, hyperuricemia and non-alcoholic fatty liver. Hyperglycemia impairs perfusion and activates redox-associated biologic pathways causing cardiac autonomic neuropathy and forming advanced glycation end products (AGEs). Atrial electrical remodeling includes shortened atrial effective refractory period (AERP) and increased AERP dispersion. Glycemic levels are independently associated with interatrial electromechanical delay. Ras homolog gene family, member A (RhoA)/ Rho associated coiled-coil forming protein kinase (ROCK) is involved in atrial fibrosis. Mitochondrial oxidative stress is possibly the main source of reactive oxygen species in diabetic atrial structural remodeling and AF perpetuation. DM upregulates Connexin 43 atrial expression and decreases phosphorylation creating hyperglycemia- associated AF substrate. Hypoglycemia has been linked to AF vulnerability by promoting fibrosis possibly through thioredoxin-interacting protein upregulation and NADPH oxidase expression [6]. Extracellular signal-regulated kinase 1/2 (ERK1/ 2), a subfamily of mitogen-activated protein kinase (MAPK), plays a role in signal transduction of atrial structural remodeling. Atrial enlargement can activate ERK pathway and induce AERP change [4]. DM related atrial fibrosis results in prolonged activation time and cycle length and local reduction of atrial electrogram voltages, thus contributing to arrhythmia [6]. Angiotensin II (AngII) has been implicated in atrial electrical remodeling characterized by shortening of the Action
Potential Duration (APD) and Effective Refractory Period (ERP) [7]. Additionally, serum concentrations of Pro-collagen type I carboxyterminal peptide (PICP), cleaved from pro-collagen during collagen I synthesis in atria, has been linked to AF prevalence [8].

Pleiotropic effects of thiazolidinediones (TZDs), ligands to the peroxisome proliferator-activated receptor gamma (PPAR $\gamma$ ), favorably affect atrial remodeling without concomitant pro-arrhythmic effects. There are two case reports of diabetic patients who experienced noteworthy improvement in paroxysmal AF following treatment with rosiglitazone [9]. According to a study comprised of 12,065 non-insulin dependent diabetic patients identified from National Health Insurance Research Database in Taiwan, TZDs independently protected diabetic patients from new onset AF with a hazard ratio of 0.69 . TZDs significantly lowered CRP by more than 45\% [3]. Pioglitazone prevented age-related AF susceptibility by improving antioxidant capacity and reducing apoptosis in a rat model. Antifibrotic effects of pioglitazone are associated with improvement of TNF- $\alpha$ and IL-6 [4]. In an in vivo rat model, pioglitazone significantly shortened atrial ERP and mean AF duration and reversed the ratio of intensity of a spin probe Carbamoyl- PROXYL to an internal standard peak, indicating recovery of age dependent reduction in antioxidation. With quantitative realtime RT-PCR, decreased mRNA expression of superoxide dismutase 2 (sod2) in the aged atria was restored. The mRNA expression levels of p22phox and gp91phox, membrane subunits of NADPH oxidase, were significantly decreased while that of Hspala, encoding HSP70, was recovered. Age related decrease in Bad phosphorylation was restored. Cleaved caspase 3 and 9, active forms in apoptotic pathway, were decreased by pioglitazone [10].

In a pressure overload $\mathrm{AF}$ rat model, pioglitazone effectively reduced atrial fibrosis through its inhibition of monocyte chemoattractant protein-1 expression [11]. In primary- cultured mouse atrial fibroblasts, pioglitazone had inhibitory effects on AngII- induced connective tissue growth factor (CTGF) mRNA and protein expression and on Transforming Growth Factor- $\beta 1$ (TGF - $\beta 1$ ) and its downstream signaling pathway. Pioglitazone pretreatment markedly suppressed Ica-L a lc subunit expression and current density amplification in HL-1 (atrial myocyte) cells. Preincubation with pioglitazone significantly inhibited AngII- induced CAMP responsive element binding protein (CREB) Ser 133 phosphorylation [12].

*Correspondence to: Yanmin Xu, International Medical School, Tianjin Medical University, No.22 Qi Xiang Tai Road, He Ping District, Tianjin 300070, P.R. China, E-mail: xuyanminphdmd@sina.com

Received: September 10, 2018; Accepted: September 24, 2018; Published: September 27, 2018 
In alloxan induced diabetic rats, rosiglitazone increased plasma SOD activity and decreased plasma malondialdehyde (MDA) levels. It reduced interatrial conduction time (IACT) and induced AF duration. Rosiglitazone attenuated the Left atrial interstitial collagen volume fraction (LACVF) index that was increased in diabetic rabbits [5]. In atrial myocytes, pioglitazone significantly inhibited AngII-induced transient outward potassium current (Ito), ultra-rapid delayed rectifier potassium (Ikur), and inward rectifier potassium current (Ik1) remodeling [7]. In $146 \mathrm{DM}$ patients with firstly identified persistent AF, PICP level along with AGE decreased significantly at 14-months of follow-up creating a possibility of reduction of permanent AF incidence in DM patient [8]. Pooled analysis of four observational studies showed a strong association between use of TZDs (pioglitazone) and risk reduction (30\%) of $\mathrm{AF}$ (new onset and recurrent). Two RCTs PROactive and RECORD failed to show beneficial effects of TZDs in preventing $\mathrm{AF}$ incidence since $\mathrm{AF}$ was reported as an adverse event instead of a predefined end-point [13].

Based on the most recent study from Liu et al.(Ref) on alloxan induced diabetic rabbits, pioglitazone minimized left atrial diameter, interventricular septal thickness and LV posterior wall thickness. It lowered AF inducibility and ameliorated increase in IACT, decrease in AERP and AERPD and APD prolongation. The mean cross sectional area (CSA) and collagen volume fraction (CVF), indices of interstitial fibrosis, increased in DM group were attenuated by pioglitazone. It also prevented expression levels of proteins involved in pro-fibrotic signalingextracellular signal-regulated kinase 2(ERK2), phosphorylation ERK (p ERK), TGF $\beta 1$, nuclear factor (NF ) $-\kappa B 50$ and HSP 70 increased in DM. Liu et al. 's study (Ref) endeavors to validate the pleiotropic role of thiazolidinediones in atrial remodeling by including diverse parameters- hemodynamics and echocardiographic measures (LVEDP, LAD, and LVEF), electrophysiology (IACT, AERP), histology (CSA, CVF) and Western blot (ERK2, TGF $\beta 1$, HSP 70) and depicting role of pioglitazone in reducing the vulnerability to AF. Reduction in electrophysiological, ion current density and protein expression changes in alloxan induced diabetic rabbits by both low $(4 \mathrm{mg} / \mathrm{kg} /$ day $)$ and high doses $(8 \mathrm{mg} / \mathrm{kg} /$ day $)$ of pioglitazone makes it an even more promising mode of upstream therapy capable of exhibiting extended anti-inflammatory, antioxidant, antifibrotic and insulin sensitizing actions. The role of other thiazolidinediones needs to be studied further and compared with pioglitazone to create a generalized class action in attenuating atrial structural remodeling chiefly responsible for $\mathrm{AF}$ perpetuation in diabetes.

In this issue of CARDIOVASCULAR THERAPY, liu et al demonstated the basic mechanism of atrial fibrillation and the information on the contribtion of Pioglitazone and make a reassuring answer to AF vulnerability in diabetics. The original research by liu et al.in this issue of CARDIOVASCULAR THERAPY makes a small step in this direction.

\section{References}

1. Staerk L, Sherer JA, Ko D, Benjamin EJ, Helm RH (2017) Atrial Fibrillation: Epidemiology, Pathophysiology, and Clinical Outcomes. Circ Res 120: 1501-1517. [Crossref]

2. Asghar O, Alam U, Hayat SA, Aghamohammadzadeh R, Heagerty AM, et al. (2013) Diabetes, obesity and atrial fibrillation: Epidemiology, mechanisms and interventions. J Atr Fibrillation 6: 47-55.

3. Chao TF, Leu HB, Huang CC (2012) Thiazolidinediones can prevent new onset atrial fibrillation in patients with non-insulin dependent diabetes. Int J Cardiol 156:199-202.

4. Liu C, Liu T, Li G (2013) Pioglitazone may offer therapeutic advantages in diabetesrelated atrial fibrillation. Int J Cardiol 168: 1603-1605. [Crossref]

5. Liu T, Zhao H, Li J, Korantzopoulos P, Li G (2014) Rosiglitazone attenuates atrial structural remodeling and atrial fibrillation promotion in alloxan-induced diabetic rabbits. Cardiovasc Ther 32:178-183

6. Goudis CA, Korantzopoulos P, Ntalas I V, Kallergis EM, Liu T, et al. (2015) Diabetes mellitus and atrial fibrillation: Pathophysiological mechanisms and potential upstream therapies. Int J Cardiol 184:617-622.

7. Liu X (2014) Pioglitazone Improves Potassium Channel Remodeling Induced by Angiotensin II in Atrial Myocytes. Med Sci Monit Basic Res 20: 153-160.

8. G LBJ (2014) Beneficial effects of pioglitazone on retardation of persistent atrial fibrillation progression in diabetes mellitus patients. Int Heart $J$ 55:499-505.

9. Liu T, Korantzopoulos P, Li G, Li J (2008) The potential role of thiazolidinediones in atrial fibrillation. Int J Cardiol 128: 129-130. [Crossref]

10. Xu D, Murakoshi N, Igarashi M, et al. (2012) PPAR-?? activator pioglitazone prevents age-related atrial fibrillation susceptibility by improving antioxidant capacity and reducing apoptosis in a rat model. J Cardiovasc Electrophysiol 23: 209-217.

11. Liu T, Li G (2012) Thiazolidinediones as novel upstream therapy for atrial fibrillation in diabetic patients: a review of current evidence. Int J Cardiol 156: 215-216. [Crossref]

12. Gu J, Liu X, Wang Q, et al. (2013) Beneficial effects of pioglitazone on atrial structural and electrical remodeling in vitro cellular models. J Mol Cell Cardiol 65:1-8.

13. Zhang Z, Zhang X, Korantzopoulos P, Letsas KP, Tse G, et al. (2017) Thiazolidinedione use and atrial fibrillation in diabetic patients: a meta-analysis. BMC Cardiovasc Disord 17: 96. [Crossref]

Copyright: (C2018 Xu Y. This is an open-access article distributed under the terms of the Creative Commons Attribution License, which permits unrestricted use, distribution, and reproduction in any medium, provided the original author and source are credited. 